\title{
Available online at www.ijrat.org
}

\section{Anti Gaussian Quadrature For Real Definite Integral}

\author{
Saumya Ranjan Jena ${ }^{1}$, Damayanti Nayak ${ }^{2}$, \\ ${ }^{1,2}$ Dept of Mathematics, School of Applied Sciences, \\ KIIT Deemed to be University, Bhubaneswar-751024, Odisha, India \\ Email: saumyafma@kiit.ac.in ${ }^{1}$, damayanti.nayak83@gmail.com ${ }^{2}$
}

\begin{abstract}
A higher degree precision quadrature rule has been constructed with the problem of determining the approximated solution of real definite integrals using anti Lobatto five point rule and anti Clenshaw Curtis seven point rule which has been further compared with another mixed quadrature rule for different integrals with their anti Gaussian rules. Some numerical examples are provided to illustrate the accuracy and comparison of absolute error of proposed rule with constituent quadrature rules.
\end{abstract}

Index Terms- Numerical Integration, Anti-Gaussian quadrature rules, Gaussian quadrature rules, Mixed quadrature rule, Degree of precision

\section{INTRODUCTION}

Numerical integration is the approximate numerical computation of an integral. Gauss quadrature could be a wide spread approach to approximate the value of an The proposed work is a comparison with [2]. They integral determined by a measure with support on the have used only the ant Gaussian with Gaussian rule for real axis. the mixed quadrature where as we have applied

Dirk P. Laurie [1] was first coined the idea of anti- Gaussian as well as anti Gaussian rule for the mixed quadrature rule. The Lobatto four point rule and Gaussian quadrature formula. The $(n+1)$ point Clenshaw Curtis five point Gaussian rule have chosen formula of anti-Gaussian quadrature rule of for derivation of Lobatto five point rule and Clenshaw degree $(2 n-1)$ integrates the polynomials of degree up Curtis seven point anti Gaussian rule each of degree of to $(2 n+1)$ with an error equal in magnitude but of to $(2 n+1)$ with an error equal in magnitude but of
opposite sign to that of the Gaussian n point formula. It $G_{w}{ }^{(n)}=\sum_{j=1}^{n} w_{j}{ }^{(n)} f\left(x_{j}{ }^{(n)}\right)$
meant the application is to evaluate the error occurred in Gaussian integration by having the distinction of degree $(2 n-1)$ for the integral between the results occurred from the two formulas. The anti Gaussian formula has positive weights and the nodes within the integration interval and reticulate by the corresponding Gaussian formula.

$I=\int_{a}^{b} f(x) w(x) d x$

The method of mixing quadrature rule is based on $G_{w}{ }^{(n)}(p)=I(p), \forall p \in P^{2 n-1} \quad$ where $P^{m}$ is the forming a higher degree precision quadrature rule by taking the convex combination of two lower precision space of polynomial of degree not greater than $m$. If quadrature rules. The concept of mixed quadrature was first introduced by Das and Pradhan [5]. Various research work have been done in this area towards the numerical evaluation of real definite integrals. Among them, Jena and Nayak [6] has applied mixed $H^{(n+1)}=\sum_{j=1}^{n+1} \lambda_{j} f\left(\zeta_{j}\right)$ is an anti Gaussian formula for $(n+1)$ point and $G^{(n)}(p)$ be $n$ point Gaussian formula, then by quadrature rule to find the approximate solution of non hypothesis $I(p)-H^{(n+1)}(p)=-\left(I(p)-G^{(n)}(p)\right)$ linear Fredholm integral equation of second kind, Jena where $p$ defined as polynomial of degree $\leq 2 n+1$. and Dash $[7]$ has established mixed quadrature over The organization of the paper is as follows. In sphere with finite element approach. Dash and section 2, construction of anti Gaussian Lobatto five Das [8],[11] has proposed identification of some point has been described. Section 3 contains Clenshaw- Curtis rule with Fejer rules and also in construction of anti Gaussian Clenshaw Curtis seven adaptive field. Tripathy etal. [9] used a mixed formed for different constituent rules in section 4 . quadrature of Lobatto four point rule with Gaussian Numerical results are verified in section 5. Section 6 quadrature for approximate evaluation of real definite has drawn some conclusion.

integrals. Singh and Dash[2] has formed a mixed 
Available online at www.ijrat.org

\section{CONSTRUCTION OF ANTI-LOBATTO FIVE POINT RULE FROM LOBATTO FOUR POINT RULE}

We choose the Lobatto four point rule,

$$
L_{w}{ }^{4}(f)=\frac{1}{6}\left[\begin{array}{l}
\{f(-1)+f(1)\}+ \\
5\left\{f\left(-\frac{1}{\sqrt{5}}\right)+f\left(\frac{1}{\sqrt{5}}\right)\right\}
\end{array}\right]
$$

to develop a five point Lobatto rule $R H_{w}{ }^{5}(f)$ from four point Lobatto rule $L_{w}{ }^{4}(f)$. Using the principle $I(p)-H^{(n+1)}(p)=-\left(I(p)-G^{(n)}(p)\right)$ as adopted in Dirk. P. Laurie [1], we obtain

$$
\begin{aligned}
& R H_{w}{ }^{5}(f)=2 \int_{-1}^{1} f(x) d x-L_{w}{ }^{4}(f) \\
& \alpha_{1} f(-1)+\alpha_{2} f\left(\xi_{1}\right)+\alpha_{3} f\left(\xi_{2}\right)+\alpha_{4} f\left(\xi_{3}\right)+ \\
& \alpha_{5} f(1)=2 \int_{-1}^{1} f(x) d x-L_{w}{ }^{4}(f)
\end{aligned}
$$

where

$$
\begin{aligned}
& R H_{w}{ }^{5}(f)=\alpha_{1} f(-1)+\alpha_{2} f\left(\xi_{1}\right)+\alpha_{3} f\left(\xi_{2}\right) \\
& +\alpha_{4} f\left(\xi_{3}\right)+\alpha_{5} f(1)
\end{aligned}
$$

In order to obtain the unknown weights and nodes, a rule of precision five has been considered. Since the rule has been integrated for polynomial of degree seven, we obtain following system of eight equations having eight unknowns namely,

$$
\begin{aligned}
& \alpha_{j}(j=1,2,3,4,5), \xi_{j}(j=1,2,3) \text { for } \\
& f(x)=x^{j}(j=0,1,2,3,4,5,6,7)
\end{aligned}
$$

The system of equations are

$\alpha_{1}+\alpha_{2}+\alpha_{3}+\alpha_{4}+\alpha_{5}=2$

$-\alpha_{1}+\alpha_{2} \xi_{1}+\alpha_{3} \xi_{2}+\alpha_{4} \xi_{3}+\alpha_{5}=0$

$-\alpha_{1}+\alpha_{2} \xi_{1}^{2}+\alpha_{3} \xi_{2}^{2}+\alpha_{4} \xi_{3}^{2}+\alpha_{5}=\frac{2}{3}$

$-\alpha_{1}+\alpha_{2} \xi_{1}^{3}+\alpha_{3} \xi_{2}^{3}+\alpha_{4} \xi_{3}^{3}+\alpha_{5}=0$

$-\alpha_{1}+\alpha_{2} \xi_{1}{ }^{4}+\alpha_{3} \xi_{2}{ }^{4}+\alpha_{4} \xi_{3}{ }^{4}+\alpha_{5}=\frac{2}{5}$

$-\alpha_{1}+\alpha_{2} \xi_{1}^{5}+\alpha_{3} \xi_{2}^{5}+\alpha_{4} \xi_{3}^{5}+\alpha_{5}=0$

$-\alpha_{1}+\alpha_{2} \xi_{1}{ }^{6}+\alpha_{3} \xi_{2}{ }^{6}+\alpha_{4} \xi_{3}{ }^{6}+\alpha_{5}=\frac{118}{525}$

$-\alpha_{1}+\alpha_{2} \xi_{1}^{7}+\alpha_{3} \xi_{2}^{7}+\alpha_{4} \xi_{3}^{7}+\alpha_{5}=0$

The solution of above system of equations are

$$
\begin{aligned}
& \alpha_{1}=\alpha_{5}=-\frac{1}{18}, \alpha_{2}=\alpha_{3}=\frac{245}{414}, \\
& \alpha_{4}=\frac{64}{69}, \xi_{1}=\sqrt{\frac{23}{35}}, \quad \xi_{2}=-\sqrt{\frac{23}{35}}, \quad \xi_{3}=0
\end{aligned}
$$

Hence the anti Lobatto five rule becomes

$$
R H_{w}{ }^{5}(f)=\left[\begin{array}{c}
\frac{245}{414}\left\{\begin{array}{l}
f\left(-\sqrt{\frac{23}{35}}\right) \\
+f\left(\sqrt{\frac{23}{35}}\right)
\end{array}\right) \\
+\frac{64}{69} f(0) \\
-\frac{1}{18}\left\{\begin{array}{l}
f(-1) \\
+f(1)
\end{array}\right.
\end{array}\right\}
$$

The error associated with the rule is computed as

$$
\begin{aligned}
& E H_{w}{ }^{5}(f)=\int_{-1}^{1} f(x) d x-R H_{w}{ }^{5}(f) \\
& =\frac{32}{525 \times 6 !} f^{v i}(0)+\frac{6208}{55125 \times 8 !} f^{v i i i}(0) \\
& +\frac{348714}{2358125 \times 10 !} f^{x}(0)+\ldots .
\end{aligned}
$$

3. CONSTRUCTION OF ANTI-CLENSHAW CURTIS SEVEN POINT RULE FROM CLENSHAW CURTIS FIVE POINT RULE

We choose the Clenshaw-Curtis five point rule,

$$
C_{w}{ }^{5}(f)=\frac{1}{15}\left[\begin{array}{l}
\{f(-1)+f(1)\}+ \\
\left\{\begin{array}{l}
f\left(-\frac{1}{\sqrt{2}}\right) \\
8 f\left(\frac{1}{\sqrt{2}}\right)
\end{array}\right\}+12 f(0)
\end{array}\right]
$$

and develop a seven point Clenshaw-Curtis rule $R H_{w}{ }^{7}(f)$ from five point Clenshaw-Curtis rule $C_{w}{ }^{5}(f)$

Using the principle $I(p)-H^{(n+1)}(p)=-\left(I(p)-G^{(n)}(p)\right)$ as adopted in Dirk. P. Laurie [1], we obtain

$$
R H_{w}{ }^{7}(f)=2 \int_{-1}^{1} f(x) d x-C_{w}{ }^{5}(f)
$$




$$
\begin{aligned}
& \alpha_{1} f(-1)+\alpha_{2} f\left(\xi_{1}\right)+\alpha_{3} f\left(\xi_{2}\right) \\
& +\alpha_{4} f\left(\xi_{3}\right)+\alpha_{5} f(1) \\
& =2 \int_{-1}^{1} f(x) d x-C_{w}{ }^{5}(f)
\end{aligned}
$$

where

$$
R H_{w}{ }^{7}(f)=\alpha_{1} f(-1)+\alpha_{2} f\left(\xi_{1}\right)
$$$$
+\alpha_{3} f\left(\xi_{2}\right)+\alpha_{4} f\left(\xi_{3}\right)+\alpha_{5} f(1)
$$

In order to obtain the unknown weights and nodes, a rule of precision five has been considered. Since the rule has been integrated for polynomial of degree seven, we obtain following system of eight equations having eight unknowns namely, $\alpha_{j}(j=1,2,3,4,5), \quad \xi_{j}(j=1,2,3)$ for $f(x)=x^{j}(j=0,1,2,3,4,5,6,7)$

The system of equations are

$\alpha_{1}+\alpha_{2}+\alpha_{3}+\alpha_{4}+\alpha_{5}=2$

$-\alpha_{1}+\alpha_{2} \xi_{1}+\alpha_{3} \xi_{2}+\alpha_{4} \xi_{3}+\alpha_{5}=0$

$-\alpha_{1}+\alpha_{2} \xi_{1}{ }^{2}+\alpha_{3} \xi_{2}{ }^{2}+\alpha_{4} \xi_{3}{ }^{2}+\alpha_{5}=\frac{2}{3}$ (3.6)

$-\alpha_{1}+\alpha_{2} \xi_{1}^{3}+\alpha_{3} \xi_{2}{ }^{3}+\alpha_{4} \xi_{3}{ }^{3}+\alpha_{5}=0$

$-\alpha_{1}+\alpha_{2} \xi_{1}^{4}+\alpha_{3} \xi_{2}{ }^{4}+\alpha_{4} \xi_{3}{ }^{4}+\alpha_{5}=\frac{2}{5}$

$-\alpha_{1}+\alpha_{2} \xi_{1}^{5}+\alpha_{3} \xi_{2}^{5}+\alpha_{4} \xi_{3}^{5}+\alpha_{5}=0$

$-\alpha_{1}+\alpha_{2} \xi_{1}{ }^{6}+\alpha_{3} \xi_{2}{ }^{6}+\alpha_{4} \xi_{3}{ }^{6}+\alpha_{5}=\frac{32}{105}$

$-\alpha_{1}+\alpha_{2} \xi_{1}{ }^{7}+\alpha_{3} \xi_{2}{ }^{7}+\alpha_{4} \xi_{3}{ }^{7}+\alpha_{5}=0$

The solution of above system of equations are $\alpha_{1}=\alpha_{5}=\frac{17}{135}, \alpha_{2}=\alpha_{3}=\frac{392}{675}$,

$\alpha_{4}=\frac{44}{75}, \xi_{1}=\sqrt{\frac{5}{14}}, \quad \xi_{2}=-\sqrt{\frac{5}{14}}, \quad \xi_{3}=0$

Hence the anti Clenshaw-Curtis seven point rule becomes

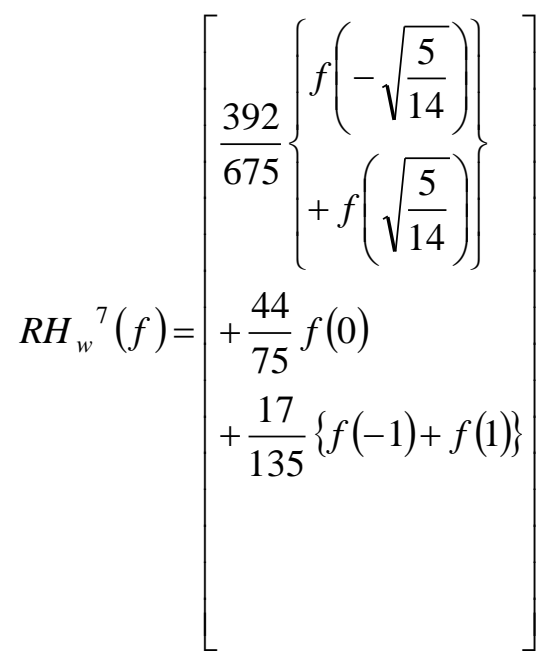

The error associated with the rule is computed as

$$
\begin{aligned}
& E H_{w}{ }^{7}(f)=\int_{-1}^{1} f(x) d x-R H_{w}{ }^{7}(f) \\
& =-\frac{2}{105 \times 6 !} f^{v i}(0)-\frac{107}{2205 \times 8 !} f^{v i i i}(0) \\
& -\frac{2897}{37730 \times 10 !} f^{x}(0) \ldots
\end{aligned}
$$

\section{CONSTRUCTION OF MIXED QUADRATURE}

RULE

In this section we have constructed a mixed quadrature rule taking four constituent rules and error analysis has been made.

4.1 Anti-Clenshaw Curtis seven point rule with anti Lobatto five point rule

We have anti-Clenshaw Curtis seven point

rule $R H_{w}{ }^{7}(f)=\left[\begin{array}{l}\frac{392}{675}\left\{f\left(-\sqrt{\frac{5}{14}}\right)+f\left(\sqrt{\frac{5}{14}}\right)\right\} \\ +\frac{44}{75} f(0)+\frac{17}{135}\{f(-1)+f(1)\}\end{array}\right]$

and the anti Lobatto five rule

$$
R H_{w}{ }^{5}(f)=\left[\begin{array}{l}
\frac{245}{414}\left\{f\left(-\sqrt{\frac{23}{35}}\right)+f\left(\sqrt{\frac{23}{35}}\right)\right\} \\
+\frac{64}{69} f(0)-\frac{1}{18}\{f(-1)+f(1)\}
\end{array}\right]
$$

where $R H_{w}{ }^{5}(f)$ and $R H_{w}{ }^{7}(f)$ is of degree of precision five and $E H_{w}{ }^{5}(f)$ and $E H_{w}{ }^{7}(f)$ denote the corresponding errors by the rules $R H_{w}{ }^{5}(f)$ and $R H_{w}{ }^{7}(f)$ for the integrals $I(f)$ respectively. Now

$$
\begin{aligned}
& I=R H_{w}{ }^{5}+E H_{w}{ }^{5} \\
& I=R H_{w}{ }^{7}+E H_{w}{ }^{7}
\end{aligned}
$$


Available online at www.ijrat.org

By Maclaurin's expansion of function in equation (4.1) and (4.2), we have

$$
\begin{aligned}
& E H_{w}{ }^{5}(f)=\frac{32}{525 \times 6 !} f^{v i}(0) \\
& +\frac{6208}{55125 \times 8 !} f^{v i i i}(0) \\
& +\frac{348714}{2358125 \times 10 !} f^{x}(0)+\ldots \\
& E H_{w}{ }^{7}(f)=-\frac{2}{105 \times 6 !} f^{v i}(0)- \\
& \frac{107}{2205 \times 8 !} f^{v i i i}(0) \\
& -\frac{2897}{37730 \times 10 !} f^{x}(0) \ldots
\end{aligned}
$$

Eliminating $f^{v i}(0)$ from

equation (4.1.3) and (4.1.4) we have

$I=\frac{1}{21}\left[5 R H_{w}{ }^{5}(f)+16 R H_{w}{ }^{7}(f)\right]$

$+\frac{1}{21}\left[5 E H_{w}{ }^{5}(f)+16 E H_{w}{ }^{7}(f)\right]$

$I(f)=R H_{w}{ }^{5} H_{w}{ }^{7}(f)+E H_{w}{ }^{5} H_{w}{ }^{7}(f)$

$R H_{w}{ }^{5} H_{w}{ }^{7}(f)=\frac{1}{21}\left[\begin{array}{l}5 R H_{w}{ }^{5}(f) \\ +16 R H_{w}{ }^{7}(f)\end{array}\right]$

which is the estimated mixed rule of precision seven. The truncated error for the approximation is

$$
\begin{aligned}
& E H_{w}{ }^{5} H_{w}{ }^{7}(f)=\frac{1}{21}\left[\begin{array}{l}
5 E H_{w}{ }^{5}(f) \\
+16 E H_{w}{ }^{7}(f)
\end{array}\right] \\
& E H_{w}{ }^{5} H_{w}{ }^{7}(f)=-\frac{112}{11025 \times 8 !} f^{v i i i}(0) \\
& -\frac{230686}{9904125 \times 10 !} f^{x}(0) \ldots
\end{aligned}
$$

4.2 Anti Lobatto five point rule with Fejer's five point second rule
We have anti Lobatto five point

$$
R H_{w}{ }^{5}(f)=\left[\begin{array}{c}
\frac{245}{414}\left\{\left(-\sqrt{\frac{23}{35}}\right)\right. \\
+f\left(\sqrt{\frac{23}{35}}\right)
\end{array}\right\}
$$

and Fejer's five point second rule

$$
R_{2 f 5}(f)=\frac{2}{45}\left[\begin{array}{l}
7\left\{f\left(-\frac{\sqrt{3}}{2}\right)+f\left(\frac{\sqrt{3}}{2}\right)\right\} \\
+9\left\{f\left(-\frac{1}{2}\right)+f\left(\frac{1}{2}\right)\right\}
\end{array}\right] \text { (4.2.2) }
$$

where the rules $R H_{w}{ }^{5}(f)$ and $R_{2 f 5}(f)$ is of precision five and $E H_{w}{ }^{5}(f)$ and $E_{2 f 5}(f)$ is the errors for the integrals $I(f)$ due to the rules $R H_{w}{ }^{5}(f)$ and $R_{2 f 5}(f)$ respectively. Now $I=R H_{w}{ }^{5}+E H_{w}{ }^{5}(f)(4.2 .1)$ $I(f)=R_{2 f 5}(f)+E_{2 f 5}(f)$

Expanding equation (4.2.1) and (4.2.2) by Maclaurin's expansion, we have

$$
\begin{aligned}
& E H_{w}{ }^{5}(f)=\frac{32}{525 \times 6 !} f^{v i}(0) \\
& +\frac{6208}{55125 \times 8 !} f^{v i i i}(0) \\
& +\frac{348714}{2358125 \times 10 !} f^{x}(0)+\ldots . \\
& E_{2 f 5}(f)=\frac{3}{280 \times 6 !} f^{v i}(0) \\
& +\frac{1}{45 \times 8 !} f^{v i i i}(0) \\
& +\frac{47}{1408 \times 10 !} f^{x}(0) \ldots
\end{aligned}
$$


Eliminating $f^{v i}(0)$ from

equation (4.2.3) and (4.2.4) we have

$$
\begin{aligned}
& I(f)=\frac{1}{1477}\left[1792 R_{2 f 5}(f)-315 R H_{w}{ }^{5}(f)\right] \\
& +\frac{1}{1477}\left[1792 E_{2 f 5}(f)-315 E H_{w}{ }^{5}(f)\right] \\
& I(f)=R H_{w}{ }^{5} R_{2 f 5}(f) \\
& +E H_{w}{ }^{5} R_{2 f 5}(f) \\
& R H_{w}{ }^{5} R_{2 f 5}(f)(f) \\
& =\frac{1}{1477}\left[\begin{array}{l}
1792 R_{2 f 5}(f)- \\
315 R H_{w}{ }^{5}(f)
\end{array}\right]
\end{aligned}
$$

which is the mixed rule of precision seven and the truncated error for this approximation is

$$
\begin{aligned}
& E H_{w}{ }^{5} R_{2 f 5}(f) \\
& =\frac{1}{1477}\left[\begin{array}{l}
1792 E_{2 f 5}(f) \\
-315 E H_{w}{ }^{5}(f)
\end{array}\right] \\
& E H_{w}{ }^{5} R_{2 f 5}(f)=\frac{6848}{2326275 \times 8 !} f^{\text {viii }}(0) \\
& +\frac{2740}{207 \times 10 !} f^{x}(0) \ldots
\end{aligned}
$$

4.3 Anti-Clenshaw Curtis seven point rule with Fejer's five point second rule

We have anti Clenshaw-Curtis seven point rule

$$
R H_{w}{ }^{7}(f)=\left[\begin{array}{l}
\frac{392}{675}\left\{f\left(-\sqrt{\frac{5}{14}}\right)+f\left(\sqrt{\frac{5}{14}}\right)\right\} \\
+\frac{44}{75} f(0)+\frac{17}{135}\{f(-1)+f(1)\}
\end{array}\right]
$$

and Fejer's five point second rule

$$
R_{2 f 5}(f)=\frac{2}{45}\left[\begin{array}{l}
7\left\{f\left(-\frac{\sqrt{3}}{2}\right)+f\left(\frac{\sqrt{3}}{2}\right)\right\} \\
+13 f(0)+9\left\{f\left(-\frac{1}{2}\right)+f\left(\frac{1}{2}\right)\right\}
\end{array}\right]
$$

Where $R H_{w}{ }^{7}(f)$ and $R_{2 f 5}(f)$ is of precision five and $E H_{w}{ }^{7}(f)$ and $E_{2 f 5}(f)$ is the error for the integrals $I(f)$ for the rules $R H_{w}{ }^{7}(f)$ and $R_{2 f 5}(f)$ respectively. Now

$$
\begin{aligned}
& I=R H_{w}{ }^{7}+E H_{w}{ }^{7} \\
& I=R_{2 f 5}+E_{2 f 5}
\end{aligned}
$$

Expanding equation (4.1) and (4.2) by Maclaurin's expansion, we have

$$
\begin{aligned}
& E H_{w}{ }^{7}(f)=-\frac{2}{105 \times 6 !} f^{v i}(0) \\
& -\frac{107}{2205 \times 8 !} f^{v i i i}(0) \quad(4.3 .3) \\
& -\frac{2897}{37730 \times 10 !} f^{x}(0) \ldots \\
& E_{2 f 5}(f)=\frac{3}{280 \times 6 !} f^{v i}(0)+ \\
& \frac{1}{45 \times 8 !} f^{v i i i}(0) \\
& +\frac{47}{1408 \times 10 !} f^{x}(0) \ldots \\
& \text { Eliminating } f^{v i}(0) \text { from } \\
& \text { equation }(4.3 .3) \text { and }(4.3 .4), \text { we have }^{v i} \\
& I=\frac{1}{175}\left[112 R_{2 f 5}(f)+63 R H_{w}{ }^{7}(f)\right] \\
& +\frac{1}{175}\left[112 E_{2 f 5}(f)+63 E H_{w}{ }^{7}(f)\right] \\
& I(f)=R H_{w}{ }^{7} R_{2 f 5}(f) \\
& +E H_{w}{ }^{7} R_{2 f 5}(f) \\
& R H_{w}{ }^{7} R_{2 f 5}(f)=\frac{1}{175}\left[112 R_{2 f 5}(f)+63 R H_{w}{ }^{7}(f)\right]
\end{aligned}
$$

which is the mixed rule of precision seven and the truncated error generated for the approximation is

$$
\begin{aligned}
& E H_{w}{ }^{7} R_{2 f 5}(f)=\frac{1}{175}\left[\begin{array}{l}
112 E_{2 f 5}(f) \\
+63 E H_{w}{ }^{7}(f)
\end{array}\right] \\
& E H_{w}{ }^{7} R_{2 f 5}(f)=-\frac{179}{55125 \times 8 !} f^{v i i i}(0) \\
& -\frac{260557}{41503000 \times 10 !} f^{x}(0) \ldots
\end{aligned}
$$

\subsection{Error Analysis and error bounds of mixed quadrature rules}

\section{Theorem-1}

Let the smooth function $f(x)$ is defined on $-1 \leq x \leq 1$, then the error $E H_{w}{ }^{5} H_{w}{ }^{7}(f)$ due to the mixed quadrature rule $R H_{w}{ }^{5} H_{w}{ }^{7}(f)$ is given by 
Available online at www.ijrat.org

$$
\begin{aligned}
& E H_{w}{ }^{5} H_{w}{ }^{7}(f)=-\frac{112}{11025 \times 8 !} f^{v i i i}(0) \\
& -\frac{230686}{9904125 \times 10 !} f^{x}(0) \ldots
\end{aligned}
$$

Proof:

The proof of theorem follows from equation (4.1.7).

Theorem-2

Let the smooth function $f(x)$ is defined on $-1 \leq x \leq 1$, then the error $E H_{w}{ }^{5} R_{2 f 5}(f)$ due to the mixed quadrature rule $R H_{w}{ }^{5} R_{2 f 5}(f)$ is given by

$$
\begin{aligned}
& E H_{w}{ }^{5} R_{2 f 5}(f)=\frac{6848}{2326275 \times 8 !} f^{\text {viii }}(0) \\
& +\frac{2740}{207 \times 10 !} f^{x}(0) \ldots
\end{aligned}
$$

\section{Proof:}

The proof of theorem follows from equation (4.2.7).

Theorem-3

Let the smooth function $f(x)$ is defined on $-1 \leq x \leq 1$, then the error $E H_{w}{ }^{7} R_{2 f 5}(f)$ due to the mixed quadrature rule $R H_{w}{ }^{7} R_{2 f 5}(f)$ is given by

$$
\begin{aligned}
& E H_{w}{ }^{7} R_{2 f 5}(f)=-\frac{179}{55125 \times 8 !} f^{v i i i}(0) \\
& -\frac{260557}{41503000 \times 10 !} f^{x}(0) \ldots
\end{aligned}
$$

\section{Proof:}

The proof of Theorem follows from equation (4.3.6).

\section{Theorem-4}

The truncated error bound for

$E H_{w}{ }^{5} H_{w}{ }^{7}(f)=I(f)-R H_{w}{ }^{5} H_{w}{ }^{7}(f)$ is

evaluated by $\left|E H_{w}{ }^{5} H_{w}{ }^{7}(f)\right| \leq \frac{64 M}{105 \times 6 !}$

where $M=\max _{-1 \leq x \leq 1}\left|f^{v i i}(x)\right|$.

\section{Proof}

$$
E H_{w}{ }^{5}(f)=\frac{32}{525 \times 6 !} f^{v i}\left(\eta_{1}\right), \quad \eta_{1} \in[-1,1]
$$$$
E H_{w}{ }^{7}(f)=-\left(\frac{2}{105 \times 6 !}\right) f^{v i}\left(\eta_{2}\right), \quad \eta_{2} \in[-1,1]
$$

$$
E H_{w}{ }^{5} H_{w}{ }^{7}(f)=\frac{32}{105 \times 6 !}\left[f^{v i}\left(\eta_{2}\right)-f^{v i}\left(\eta_{1}\right)\right]
$$

(Conte \& Boor $[10]$ )

$$
\begin{aligned}
& \left|E H_{w}{ }^{5} H_{w}{ }^{7}(f)\right| \cong \frac{32}{105 \times 6 !}\left[f^{v i}(d)-f^{v i}(c)\right] \\
& =\frac{32}{105 \times 6 !} \int_{-1}^{1} f^{v i i}(x) d x \\
& =\frac{32}{105 \times 6 !}(d-c) f^{v i i}(\gamma) \\
& \quad \text { or } \\
& \text { some } \gamma \in[-1,1] \\
& \text { where }|d-c| \leq 2 \\
& \left|E H_{w}{ }^{5} H_{w}{ }^{7}(f)\right| \leq \frac{64}{105 \times 6 !} f^{v i i}(\gamma)
\end{aligned}
$$$$
\text { where }|d-c| \leq 2
$$

Hence $\left|E H_{w}{ }^{5} H_{w}{ }^{7}(f)\right| \leq \frac{64 M}{105 \times 6 !}$ where

$M=\max _{-1 \leq x \leq 1}\left|f^{v i i}(x)\right|$.

\section{Theorem-5}

The truncated error bound for

$$
\begin{aligned}
& E H_{w}{ }^{5} R_{2 f 5}(f)=I(f)-R H_{w}{ }^{5} R_{2 f 5}(f) \text { is } \\
& \text { evaluated by }\left|E H_{w}{ }^{5} R_{2 f 5}(f)\right| \leq \frac{1344 M}{51695 \times 6 !} \\
& \text { where } M=\max _{-1 \leq x \leq 1}\left|f^{v i i}(x)\right|
\end{aligned}
$$

Proof:

$$
\begin{array}{ll}
E H_{w}{ }^{5}(f)=\frac{32}{525 \times 6 !} f^{v i}\left(\eta_{1}\right), & \eta_{1} \in[-1,1] \\
E R_{2 f 5}(f)=\frac{3}{280 \times 6 !} f^{v i}\left(\eta_{2}\right), & \eta_{2} \in[-1,1] \\
E H_{w}{ }^{5} R_{2 f 5}(f)=\frac{672}{51695 \times 6 !}\left[f^{v i}\left(\eta_{2}\right)-f^{v i}\left(\eta_{1}\right)\right]
\end{array}
$$

As per Theorem-4,

$$
\begin{aligned}
& \left|E H_{w}{ }^{5} R_{2 f 5}(f)\right| \cong \frac{672}{51695 \times 6 !}\left[f^{v i}(d)-f^{v i}(c)\right] \\
& =\frac{672}{51695 \times 6 !} \int_{-1}^{1} f^{v i i}(x) d x \\
& =\frac{672}{51695 \times 6 !}(d-c) f^{v i i}(\gamma) \\
& \text { for some } \gamma \in[-1,1]
\end{aligned}
$$




\section{Available online at www.ijrat.org}

where $|d-c| \leq 2$

$\left|E H_{w}{ }^{5} R_{2 f 5}(f)\right| \leq \frac{1344}{51695 \times 6 !} f^{v i i}(\gamma)$

Hence $\left|E H_{w}{ }^{5} R_{2 f 5}(f)\right| \leq \frac{1344 M}{51695 \times 6 \text { ! }}$

where $M=\max _{-1 \leq x \leq 1}\left|f^{v i i}(x)\right|$.

\section{Theorem-6}

The truncated error bound for

$E H_{w}{ }^{7} R_{2 f 5}(f)=I(f)-R H_{w}{ }^{7} R_{2 f 5}(f)$ is

evaluated by $\left|E H_{w}{ }^{7} R_{2 f 5}(f)\right| \leq \frac{84 M}{6125 \times 6 \text { ! }}$

where $M=\max _{-1 \leq x \leq 1}\left|f^{v i i}(x)\right|$

Proof

$\begin{array}{ll}E H_{w}{ }^{7}(f)=-\frac{2}{105 \times 6 !} f^{v i}\left(\eta_{1}\right), & \eta_{1} \in[-1,1] \\ E R_{2 f 5}(f)=\frac{3}{280 \times 6 !} f^{v i}\left(\eta_{2}\right), & \eta_{2} \in[-1,1]\end{array}$

$E H_{w}{ }^{7} R_{2 f 5}(f)=\frac{42}{6125 \times 6 !}\left[f^{v i}\left(\eta_{2}\right)-f^{v i}\left(\eta_{1}\right)\right]$

As per Theorem-4 and Theorem-5,

$\left|E H_{w}{ }^{7} R_{2 f 5}(f)\right| \cong \frac{42}{6125 \times 6 !}\left[f^{v i}(d)-f^{v i}(c)\right]$

$=\frac{42}{6125 \times 6 !} \int_{-1}^{1} f^{v i i}(x) d x=\frac{42}{6125 \times 6 !}(d-c) f^{v i i}(\gamma$

forsome $\gamma \in[-1,1]$

where $|d-c| \leq 2$

$\left|E H_{w}{ }^{7} R_{2 f 5}(f)\right| \leq \frac{84}{6125 \times 6 !} f^{v i i}(\gamma)$

Hence $\left|E H_{w}{ }^{7} R_{2 f 5}(f)\right| \leq \frac{84 M}{6125 \times 6 \text { ! }}$

where $M=\max _{-1 \leq x \leq 1}\left|f^{v i i}(x)\right|$.

5. Numerical Results

In this section some numerical examples are taken to validate our proposed work. The absolute error shows a solid comparison between different mixed quadrature rules $\left(R_{H w^{5} \mathrm{Hw}^{7}}(f)\right), \quad\left(R_{H w^{5} 2 f 5}(f)\right)$ $\left(R_{H w^{5} 2 f 5}(f)\right)$ which provides better approximation to exact results than the constituent rules for different integrals.

The approximate value of the following integrals have been calculated which has given in Table-1.

$I_{1}=\int_{-1}^{1} e^{x} d x=2.350402387287603, I_{2}=\int_{0}^{1} e^{x^{2}} d x=1$ .462651745907181

$I_{3}=\int_{0}^{1} e^{-x^{2}} d x=0.746824132812427$,

$I_{4}=\int_{1}^{3} \frac{\sin ^{2} x}{x} d x=0.794825180668111$,

$I_{5}=\int_{0}^{1} \sqrt{x} d x=0.6666666666666667$,

$I_{6}=\int_{0}^{1} \sqrt{x} \sin x d x=0.364221932032132$,

$I_{7}=\int_{0}^{1} \sin \sqrt{\pi x} d x=0.849726325420498$,

$I_{8}=\int_{0}^{1} x^{10} \cos x^{16} d x=0.049121729517639$

$I_{9}=\int_{2}^{3} \frac{\ln \sqrt{x}}{x} d x=0.181623986723595$,

$I_{10}=\int_{1}^{2} \frac{\cosh x}{\sqrt{x}} d x=1.980270250563978$,

$I_{11}=\int_{3}^{4} \cosh ^{-1} x \log x d x=2.40709642933493$

Table-1(Comparison of absolute error for anti mixed quadrature rule $\left(R_{\mathrm{Hw}^{5} \mathrm{Hw}^{7}}(f)\right)$ with mixed rule of Gaussian and anti Gaussian $\left(R_{H w^{5} 2 f 5}(f)\right)$ and $\left(R_{H w^{5} 2 f 5}(f)\right)$ ).

\begin{tabular}{|c|c|c|c|c|c|c|}
\hline$I$ & $\begin{array}{l}E_{H w^{5}} \\
(f)\end{array}$ & $\begin{array}{l}E_{2 f 5} \\
(f)\end{array}$ & $\begin{array}{l}E_{H w^{7}} \\
(f)\end{array}$ & $\begin{array}{l}E_{H w^{5} H} \\
(f)\end{array}$ & $\begin{array}{l}E_{H w^{5} 2 f 5} \\
(f)\end{array}$ & $\begin{array}{l}E_{H w^{7}} \\
(f)\end{array}$ \\
\hline$I_{1}$ & $\begin{array}{l}0.00 \\
0087 \\
4902 \\
6950 \\
3\end{array}$ & $\begin{array}{l}0.000 \\
01544 \\
13877 \\
45\end{array}$ & $\begin{array}{l}0.000 \\
02767 \\
99203 \\
75\end{array}$ & $\begin{array}{l}0.000 \\
00025 \\
84465 \\
95\end{array}$ & $\begin{array}{l}0.0000 \\
00075 \\
51248 \\
8\end{array}$ & $\begin{array}{l}0.00 \\
0000 \\
0822 \\
8317 \\
9\end{array}$ \\
\hline${ }^{d} I_{2}$ & $\begin{array}{l}0.00 \\
0326 \\
3044 \\
\end{array}$ & $\begin{array}{l}0.000 \\
05844 \\
11378 \\
\end{array}$ & $\begin{array}{l}0.000 \\
10765 \\
89892 \\
\end{array}$ & $\begin{array}{l}0.000 \\
00433 \\
43685 \\
\end{array}$ & $\begin{array}{l}0.0000 \\
01313 \\
89805 \\
\end{array}$ & $\begin{array}{l}0.00 \\
0001 \\
3549 \\
\end{array}$ \\
\hline
\end{tabular}


Available online at www.ijrat.org

\begin{tabular}{|c|c|c|c|c|c|c|}
\hline & $\begin{array}{l}1788 \\
4\end{array}$ & 70 & 45 & 00 & 6 & $\begin{array}{l}0789 \\
1\end{array}$ \\
\hline \multirow{5}{*}{$I_{3}$} & 0.00 & 0.000 & 0.000 & 0.000 & 0.0000 & 0.00 \\
\hline & 0012 & 00213 & 00352 & 00029 & 00079 & 0000 \\
\hline & 4956 & 05773 & 16722 & 19767 & 98397 & 0957 \\
\hline & 5367 & 28 & 35 & 91 & 8 & 6748 \\
\hline & 3 & & & & & 5 \\
\hline \multirow{5}{*}{$I$} & 0.00 & 0.000 & 0.000 & 0.000 & 0.0000 & 0.00 \\
\hline & 0227 & 03939 & 06754 & 00276 & 00776 & 0000 \\
\hline & 7650 & 70923 & 47352 & 71151 & 16597 & 8980 \\
\hline & 3667 & 07 & 97 & 71 & 0 & 3437 \\
\hline & 4 & & & & & 0 \\
\hline \multirow{5}{*}{$I_{5}$} & 0.00 & 0.001 & 0.006 & 0.003 & 0.0003 & 0.00 \\
\hline & 6073 & 32957 & 52000 & 52160 & 17887 & 1496 \\
\hline & 2674 & 53161 & 50195 & 68133 & 42099 & 2736 \\
\hline & 4668 & 37 & 92 & 35 & 6 & 0425 \\
\hline & 6 & & & & & \\
\hline \multirow{5}{*}{$I_{6}$} & 0.00 & 0.000 & 0.000 & 0.000 & 0.0000 & 0.00 \\
\hline & 0304 & 07092 & 18232 & 06639 & 21089 & 0020 \\
\hline & 5806 & 18388 & 66554 & 63559 & 40112 & 2476 \\
\hline & 0249 & 63 & 72 & 6 & 1 & 1909 \\
\hline & 6 & & & & & 8 \\
\hline \multirow{5}{*}{$I_{7}$} & 0.01 & 0.002 & 0.011 & 0.006 & 0.0005 & 0.00 \\
\hline & 1050 & 42791 & 73085 & 30457 & 83410 & 2671 \\
\hline & 1526 & 52683 & 02728 & 00702 & 48677 & 1603 \\
\hline & 5770 & 85 & 01 & 98 & 6 & 2644 \\
\hline & 5 & & & & & 2 \\
\hline \multirow{5}{*}{$I_{8}$} & 0.00 & 0.001 & 0.007 & 0.006 & 0.0028 & 0.00 \\
\hline & 5114 & 41956 & 12085 & 64312 & 13060 & 5836 \\
\hline & 3904 & 30211 & 61627 & 62418 & 21653 & 7181 \\
\hline & 9503 & 41 & 05 & 31 & 4 & 3539 \\
\hline & 6 & & & & & 7 \\
\hline \multirow{5}{*}{$I_{9}$} & 0.00 & 0.000 & 0.000 & 0.000 & 0.0000 & 0.00 \\
\hline & 0000 & 00011 & 00021 & 00000 & 00001 & 0000 \\
\hline & 6534 & 61459 & 10239 & 51994 & 55744 & 0016 \\
\hline & 3881 & 68 & 53 & 85 & 7 & 3520 \\
\hline & 2 & & & & & 3 \\
\hline \multirow{5}{*}{$I_{1}$} & 0.00 & 0.000 & 0.000 & 0.000 & 0.0000 & 0.00 \\
\hline & 0010 & 00182 & 00338 & 00016 & 00050 & 0000 \\
\hline & 1431 & 42603 & 06413 & 06839 & 08316 & 0495 \\
\hline & 798 & 13 & 78 & 46 & 4 & 0429 \\
\hline & & & & & & 6 \\
\hline \multirow{4}{*}{$I_{1}$} & 0.00 & 0.000 & 0.000 & 0.000 & 0.0000 & 0.00 \\
\hline & 0000 & 00001 & 00002 & 00000 & 00000 & 0000 \\
\hline & 0691 & 22301 & 20113 & 03056 & 09022 & 0000 \\
\hline & $\begin{array}{l}5276 \\
5\end{array}$ & 25 & 93 & 41 & 5 & 9682 \\
\hline
\end{tabular}

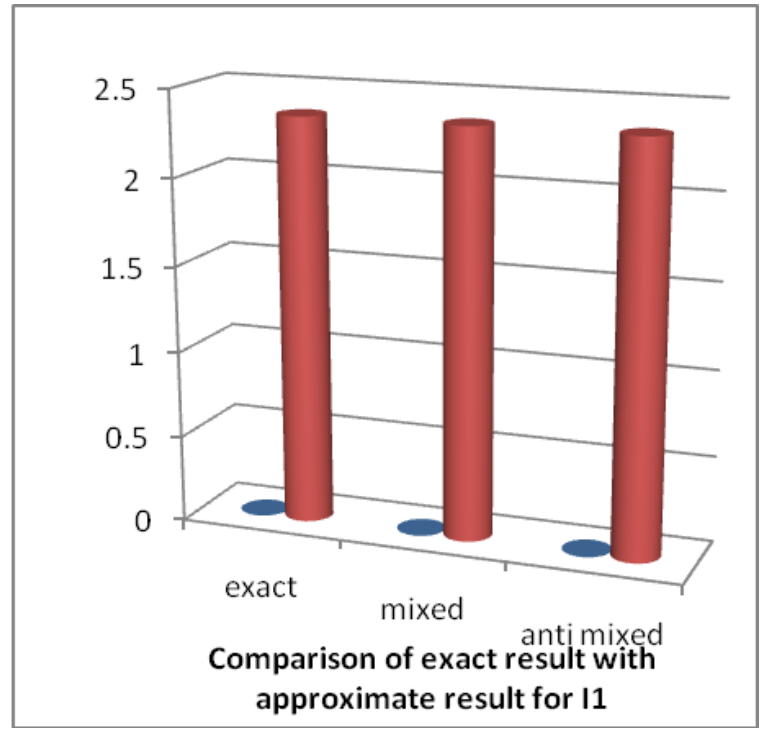

(Fig.-1)

\section{CONCLUSION}

The efficiency of our proposed rule is a good agreement with the exact result, which has been drawn from Table-1 numerically as well as from FIG-1 graphically. Error analysis of these methods besides the test numerical examples provide a solid foundation to compare between anti Gaussian-anti Gaussian mixed quadrature rule and mixed Gaussian-anti Gaussian quadrature rule for numerical estimation of real definite integrals. The main advantages of the presented method is its simple computational evaluations which is wholly competitive in comparison with the Gaussian methods.

\section{REFERENCES}

[1] P.Dirk.Laurie(1996), "Anti Gaussian quadrature formulas", Mathematics of computation, 65,739749.

[2] B.P. Singh and R. B. Dash,(2016) "Forming a mixed quadrature rule using an anti-Lobatto four point quadrature rule", Journal of Progressive Research in Mathematics 7(3), 1092-1101.

[3] Kendall.E.Atkinson (1989). “An introduction to numerical analysis", second edition, John Wiley and Sons

[4] R.N. Das and G. Pradhan, (1996) "A mixed quadrature rule for approximate evaluation of real definite integrals"Int.J.Math.E du.Sci.Technol.,27(1996),279-283.

[5] J.P. Davis and P. Rabinowitz, (1984) "Method of numerical integration", second ed. Academic press Inc.san Diego

[6] S. Jena and D. Nayak (2015) "Hybrid quadrature for numerical treatment of nonlinear Fredholm Integral 
International Journal of Research in Advent Technology, Vol.7, No.5, May 2019

E-ISSN: 2321-9637

Available online at www.ijrat.org

equation with separable kernel”,Int. J. Appl. Math. Stat., 53(4), 83-89.

[7] S. Jena and P. Dash (2014)“Approximaton of real definite integrals via hybrid quadrature domain" Int. J. Sci. Engg. Tech. Res.3(12),3188-3191.

[8] R B. Dash and D, Das(2011) "Identification of some clenshaw- Curtis quadrature rule as mixed quadrature of Fejer and Newton-Cote type of rules", Int. J. of Mathematical Sciences and Applications, 1(3), 1493-1496.

[9] A.K. Tripathy, R. B Dash. and A..Baral,( 2015)“A mixed quadrature rule blending Lobatto and Gauss Legendre three point rule for approximate evaluation of real definite integrals",Journal of computing science and Mathematics, 6(4),366-377.

[10] S. Conte and C. De. Boor, (1980)“Elementary Numerical Analysis", Tata Mac-Graw Hill,

[11] R. B. Dash and D. Das,(2011) A mixed quadrature rule by blending Clenshaw-Curtis and Gauss Legendre quadrature rules for approximation of real definite integrals in adaptive environment, Proceedings of the International multi conference of Engineers and computer scientists, 1,202-205 